Max-Planck-Institut für demografische Forschung

Max Planck Institute for Demographic Research

Konrad-Zuse-Strasse 1 - D-18057 Rostock · GERMANY

Tel +49 (0) 3812081 - 0; Fax +49 (0) 3812081 - 202;

http://www.demogr.mpg.de

MPIDR WORKING PAPER WP 2008-016

APRIL 2008

\title{
Exogenous Determinants of Early-Life \\ Conditions, and Mortality Later in Life
}

Gerard J. van den Berg (gberg@feweb.vu.nl)

Gabriele Doblhammer-Reiter (doblhammer@demogr.mpg.de)

Kaare Christensen (kchristensen@health.sdu.dk)

(C) Copyright is held by the authors.

Working papers of the Max Planck Institute for Demographic Research receive only limited review. Views or opinions expressed in working papers are attributable to the authors and do not necessarily reflect those of the Institute. 


\title{
Exogenous Determinants of Early-Life Conditions, and Mortality Later in Life
}

\author{
Gerard J. van den Berg* \\ Gabriele Doblhammer-Reiter** \\ Kaare Christensen***
}

\begin{abstract}
* VU University Amsterdam, IFAU-Uppsala, Netspar, CEPR, IZA. Address: Department of Economics, Free University Amsterdam, De Boelelaan 1105, 1081 HV Amsterdam, The Netherlands.

** University of Rostock, and Max Planck Institute for Demographic Research *** University of Southern Denmark, the Danish Twin Registry, and The Danish Aging Research Center.
\end{abstract}

Date: March 2008

Keywords: longevity, birth, health, business cycle, recession, life expectancy, lifetimes, developmental origins.

\section{Acknowledgements:}

We thank participants in the IUSSP Seminar "Early-life conditions, social mobility and other factors that influence survival to old age"' for very helpful comments. We also thank Axel Skytthe for help with the Danish twin registry data and Ingrid Henriksen, Mette Bjarnholt and Mette Erjnaes for help with the Danish historical time series data. We acknowledge financial support from the Max Planck Institute for Demographic Research. 


\section{Abstract}

We analyze causal effects of conditions early in life on the individual mortality rate later in life. Conditions early in life are captured by transitory features of the macro environment around birth, notably the state of the business cycle around birth, but also food price deviations, weather indicators, and demographic indicators. We argue that these features can only affect high-age mortality by way of the individual early-life conditions. Moreover, they are exogenous from the individual point of view, which is a methodological advantage compared to the use of unique characteristics of the newborn individual or his family or household as early-life indicators. We collected national annual time-series data on the above-mentioned indicators, and we combine these to the individual data records from the Danish Twin Registry covering births in 1873-1906. The empirical analyses (mostly based on the estimation of duration models) indicate a significant negative causal effect of economic conditions early in life on individual mortality rates at higher ages. If the national economic performance in the year of birth exceeds its trend value (i.e., if the business cycle is favorable) then the mortality rate later in life is lower. The implied effect on the median lifetime of those who survive until age 35 is about 10 months. A systematic empirical exploration of all macro indicators reveals that economic conditions in the first years after birth also affect mortality rates later in life. 


\section{Introduction}

This study analyzes the effects of early-life conditions (economic, nutritional, meteorological, in terms of disease exposure, and otherwise) on mortality rates later in life. Recently, there has been a growing interest in the importance of conditions early in life on health and mortality outcomes later in life. Knowledge on the magnitude of such long run effects may have policy implications. If being born under certain adverse conditions increases the individual mortality rate in the long run (and therefore has a negative effect on longevity) then the value of life is reduced for those affected, and this would increase the benefits of supportive policies for such groups of individuals. The long-run effect of early-life conditions on the mortality rate may be smaller than the instantaneous effect of current conditions, but the former exert their influence over a longer time span, and they are more amenable to preventive intervention between infancy and the manifestation of the effect.

The analysis of the effects of childhood conditions on later mortality is hampered by strong data requirements and methodological difficulties. First, the data need to have a sufficiently long time span. Secondly, the indicators of childhood conditions must be exogenous for health and mortality later in life. For example, earnings of the parents are to some extent determined by unobserved factors that also directly affect the health of individuals at higher ages, and these are therefore less suitable as indicators. More generally, individual variation in childhood conditions and later health and mortality 
outcomes may be jointly affected by unobserved heterogeneity, leading to simultaneity bias.

Some recent studies avoid these methodological problems by using data on indicators of individual conditions early in life with the property that the only way in which the indicator can plausibly affect high-age mortality is by way of the individual early-life conditions. By analogy to the econometrics literature, these may be called instrumental variables. Such indicators do not give rise to endogeneity and simultaneity biases, because they are exogenous from the individual point of view.

Well-known examples are provided by natural experiments. The month of birth has been recently used to study the effects of nutrition and disease exposure on mortality later in life (see e.g. the survey in Doblhammer, 2004). Other studies use epidemics, wars, or famines, as instruments for conditions in utero and early in life. The results are inconclusive (e.g. Kannisto, Christensen \& Vaupel, 1997; Almond, 2002, Sparén, Vågerö, Shestov, Plavinskaja, Parfenova, Hoptiar et al., 2004, Lumey, Stein, Kahn, Van der Pal-De Bruin, Blauw, Zybert et al., 2007). One reason may be that famines affect a range of socioeconomic outcomes: Stein, Susser, Saenger \& Marolla (1975) showed that during the Dutch hunger winter 1944-1945 fertility was comparatively higher among groups of higher socioeconomic status. Such selective fertility may counterbalance the negative long-term effects of the famine.

An alternative approach exploiting exogenous variation was pioneered by Bengtsson \& Lindström (2000). In that study, the transitory component (or deviation) in the price of rye around birth is used as an indicator of food accessibility early in life, and any 
observed relation between this indicator and the mortality rate later in life signifies the existence of a long-run causal effect of food accessibility on mortality later in life. Similarly, the transitory component in the local infant mortality rate is used as an indicator of exposure to diseases early in life (see also subsequent applications by Bengtsson \& Lindström, 2003). These studies use data from a relatively small area in Sweden. Catalano \& Bruckner (2006a) study the relation between the lifespan of children who survived to age five and mortality in the same cohort up to age five, in $19^{\text {th }}$ century birth cohorts in Sweden, Denmark, and England and Wales. Van den Berg, Lindeboom \& Portrait (2006) use the state of the business cycle at early ages as determinants of individual mortality. Cyclical macro-economic conditions during pregnancy of the mother and childhood may have effects on mortality later in life because they are unanticipated and affect household income. In a recession, the provision of sufficient nutrients and good living conditions for children and pregnant women may be hampered. Van den Berg et al. (2006) find that the average lifetime duration in the Netherlands in the 19th century is reduced if the individual is born in a recession as compared to being born in a boom, under otherwise identical conditions during life. Note that cyclical fluctuations of macro conditions are less dramatic than the extreme shocks generated by famines and epidemics. Indeed, Van den Berg, Lindeboom \& López (2007) show that the distribution of socio-economic status among parents of newborns does not depend on the business cycle at or around birth.

In the present paper we aim to advance on the literature by way of a systematic analysis of the effects of cyclical fluctuations and transitory components in macro conditions around the birth date on individual mortality later in life. We examine whether the above- 
mentioned results on the long-run effect of the business cycle early in life in the Netherlands are confirmed by data from Denmark. Subsequently, we examine whether the business-cycle effects can be understood in terms of other macro-level fluctuations. We also examine the importance of the timing of the macro fluctuations around the date of birth.

The analysis uses the Danish Twin Registry Data. An advantage of this data set, as compared to the national statistics as collected in e.g. the Human Mortality Database, is that the exact dates of birth and death are observed for individuals born as early as in the 1870s (compare e.g. Andreev, 2002), and that some personal characteristics are collected. We merge these data with historical information on macro-level exogenous indicators of early life conditions. These consist of some sets of variables measured at annual rates. First, we derive transitory economic indicators for each of the following variables: GDP, inflation, the average wage rate, the unemployment rate, and food prices of a range of products. Secondly, we use meteorological indicators, notably on cloud cover, temperature, and rainfall. (For example, Moore, Cole, Poskitt, Sonko, Whitehead, McGregor et al., 1997, find a long-run effect of being born in the wet season in Gambia on mortality later in life.) Thirdly, we use demographic indicators, notably infant mortality rates and sex ratios among newborns, and we also examine the season of birth. The sex ratio is an indicator of the amount of stress to which potential prospective mothers are exposed. For example, Catalano, Bruckner, Hartig \& Ong (2005) find a significant inverse relation between the sex ratio among newborns and the use of antidepressants among women in the population. It has also been found that male cohorts born in years with a high sex ratio experience lower life expectancy (Catalano \& 
Bruckner, 2006b). This could be interpreted as the effect of selection (see the discussion in Subsection 3.1).

Most of our indicators are annual and at a national level. By comparing the results to those in the literature, our analysis provides insight into the extent to which these indicators are sufficiently informative on individual early-life conditions. For example, it is not clear whether annual national average food prices capture early-life conditions. More in general, our results provide additional insights into the relevance of early life conditions for mortality later in life.

As noted above, long-run effects may suggest policy interventions. For example, if being born in a recession increases the individual mortality rate in the long run then it may be sensible to target policy at infants born in recessions (see however below for a discussion of evidence on period effects of recessions). Their mortality later in life could be significantly reduced if their conditions are improved upon, for example by monitoring their health shortly after birth and by providing food, housing, and health care. We should point out that living conditions in Denmark around 1900 were relatively good in comparison to most other countries at the time and in comparison to many developing countries today. Health insurance coverage was high. Denmark arguably had the best health care system in the world in terms of well-being of mothers and infants (see Løkke, 2007, for a detailed survey). Insurance societies paid out sickness absence benefits to employed workers who had fallen ill. In general, there was an extensive poor relief system. For current developing countries, which in certain aspects (like living and economic conditions) could be regarded as similar to Denmark in the period evaluated in 
the present paper, the existing literature has focused on inequalities in infant and child mortality by household socioeconomic status, since there are typically no long run data registers (see Sastry, 2004). In this sense, our paper aims to complement these studies by studying long run mortality effects.

The Danish twin data have been used by many other studies. Christensen, Vaupel, Holm \& Yashin (1995) and Christensen, Wienke, Skytthe, Holm, Vaupel \& Yashin (2001) compare patterns of mortality across age and cohort intervals in the twin data to the corresponding intervals in the general population, and they conclude that the patterns are usually the same for adults. This suggests that twins are not necessarily different from single births when it comes to the mortality distribution at higher ages, which supports the relevance of our analyses. Note that at the household level, a twin birth itself may lead to more economic hardships. To the extent that this is more relevant if birth takes place during adverse conditions, twin data would display stronger long-run effects than data on singletons.

Our focus on long-run effects of economic fluctuations is somewhat distinct from the focus in the expanding body of literature about the period effects of economic micro and macro conditions on mortality (for an extensive overview based on historical data see Bengtsson, Campbell \& Lee 2004). The general conclusion is that period mortality responded systematically to short-term variations in prices and wages (Lee, Campbell \& Bengtsson, 2004) and is graduated by age, gender, family and community preferences and resources. For contemporary populations, Ruhm (2000) shows that period mortality varies procyclically with economic conditions, in particular among younger adults. The 
most likely explanations are changes in obesity, smoking, diet and exercise, with unhealthy life styles increasing during prosperous years. There is also evidence, however, that sustained economic growth may improve health, while short-lasting expansions may worsen it . This is supported by Catalano (2002) who shows that increases in health have contributed to the decreasing mortality among the oldest old during the last century. In any case, it is not obvious and it probably depends much on the social and cultural environment whether the youngest or the oldest suffer most during bad times (Campbell et al., 2004).

The paper is organized as follows. Section 2 presents the data and discusses variables that we use in the analyses. Section 3 provides descriptive analyses and the empirical results. Section 4 concludes.

\section{The data}

\subsection{Individual records from the Twin Registry}

Our data are derived from the Danish Twin Registry. We refer to studies listed in Section 1 for detailed descriptions of the registry. We have access to a sample of all same-sex twins in the registry who were born in the years 1870-1930 (as we explain below, we mostly restrict attention to birth years 1873-1906). In addition, for each twin pair, both twins have to be alive on January 1, 1943. The individual lifetime durations are observed in days. The observation window ends on January 6, 2004, so individuals still alive then 
have right-censored durations. Figure 1 gives the Lexis diagram of the lifetime observations. The data also provide the gender and the zygosity (if observed), and information on the marriage date and marital status at death. When we select explanatory variables for individual mortality from these data, we restrict attention to characteristics that are realized at birth as opposed to later in life, for the reason that the latter may be endogenous or confounded. In particular, we do not include variables on life events like marriage.

Most of our analyses concern those born in the period before 1907. This is for two reasons. First, and most importantly, the mortality determinants among those born in later years may be systematically different because the increasing welfare in later years led to a dampening of the effect of a recession and other economic hardships on a household's food provision. In particular, in 1907, unemployment benefits were introduced in Denmark. A second reason for separate analyses on those born before 1907 is that many of the individuals born after 1906 are still alive at the end of the observation window. For example, among the about 3500 individuals born in $1925-1930$, as many as $59 \%$ have right-censored lifetime durations. In the sample, the number of individuals per birth year increases strongly with the birth year, so adding some cohorts born shortly after 1907 to the cohorts born before 1907 results in a sample in which the later-born cohorts dominate. The estimation results are not sensitive with respect to the cut-off year. 


\subsection{Data on macro-economic outcomes, meteorological conditions, and}

\section{demographic indicators}

As noted in the introduction, we use a range of variables to capture exogenous indicators of early life conditions. To explain our methodology, consider the historical time-series data on the national annual per-capita gross domestic product (GDP) in constant prices. To capture the long-run effects of conditions early in life, one might want to compare an individual born in a good era to an otherwise identical individual born in a bad era, following the line of thought that a high GDP goes along with better individual economic conditions for many individuals. However, this approach is uninformative on effects of individual early life conditions, due to the steady secular improvements in life conditions over time. After all, a prolonged era with a high GDP also leads to innovation and investment in hygiene and health care, which decreases mortality later in life for those born in this era. A related practical complication is that GDP displays a strong positive trend over time. A high GDP level at birth implies a high GDP level throughout life. An empirical analysis that tries to take this into account by allowing the mortality rate at a given age to depend on current and past GDP levels leads to estimates that are potentially very sensitive to small model misspecifications. For example, if the postulated relation is log-linear in the mortality rate and current GDP, and the true relation is slightly different, then this may show up as a significant effect of GDP earlier in life.

Now consider the effects of short-term cyclical movements in GDP. Contrary to the effect of the trend value or current level of GDP, cyclical effects are not affected by secular improvements. At the individual level, cyclical changes often involve unexpected 
income fluctuations. So as an alternative approach one could try to compare cohorts born in booms to those born in recessions, with otherwise identical circumstances throughout life. Still, due to the secular improvements over time, being born in different stages of the cycle entails that the individuals lead their lives under somewhat different current conditions at each age. As a conservative strategy one may compare a cohort born in a boom to the cohort born in the subsequent recession, because the latter benefit more from secular developments than the former, so that an observed decrease of expected lifetimes can be attributed to the cyclical effect. More in general, one may relate the mortality rate later in life to the state of the business cycle early in life for many different birth cohorts. To proceed one therefore needs to assign a value of a cyclical indicator to each year.

For GDP, the analysis below is based on a trend/cycle decomposition of log annual real per-capita GDP using the Hodrick-Prescott (HP) filter. It is important that to ensure that the time series of the cyclical component (or deviation) of GDP does not display a trend over the interval of birth years that we consider. If it would have such a trend then this may influence the estimated effect of the cyclical component at birth on mortality later in life. This is related to the discussion earlier in this subsection. The mean lifetime duration tends to increase over time. If the cyclical component has a positive trend in the birth year interval then the estimation routine may incorrectly interpret this as evidence that the cyclical component at birth has a positive long run effect on longevity. In general, to prevent a trend, one has to apply a separate filter to the subperiod considered, or one has to use a relatively low smoothing parameter. For GDP we use a smoothing parameter 100 which is low. In fact, the values of the cyclical terms are very robust with respect to the actual decomposition method and smoothing parameter, and so are the resulting intervals 
within which the terms are positive or negative. We are therefore in the fortunate position that booms and recessions are clearly identifiable in the data. Moreover, the empirical analysis provides virtually identical results if other decomposition methods are used. Due to the large number of years between birth and the observation window for deaths, we do not need to be concerned about correlations between fluctuations in early-life conditions and period fluctuations in mortality rates later in life.

The raw GDP data are from Mitchell (2003). To calculate GDP in real terms we need to deflate the nominal time series. ${ }^{1}$ For this we use the price index series of Johansen (1985) and Mitchell (2003). The same series is used to construct a time series of annual inflation for all years until 2004. The GDP data for the years 1870-1872 seem unreliable. The decomposition gives extremely high values of the cyclical deviation of GDP. We therefore omit these years from the analysis. Figure 2 displays the cycle and trend as functions of calendar time. Time-series analyses show that our cyclical indicator displays some positive autocorrelation with a lag of two years. This makes it useful to estimate models with cyclical deviations at ages 1 up to 4 as well.

We carried out an extensive search for prices of food products in Denmark. For the period 1873-1906 we have been able to construct uninterrupted series of national annual average trade prices of wheat, rye, barley, and bacon. These data are from Christensen (1985) and Statistics Denmark (1958). Wheat in particular was a major ingredient of food intake. Moreover, it was mostly imported, so that a high price should be unambiguously disadvantageous. Barley was mostly exported, so that a high price can be expected to be beneficial for the sub-population of barley farmers. In fact, the price deviations for the 
three cereals display great similarities, and their correlations in the period 1873-1906 are in the range of $0.65-0.75$. The correlations to the bacon price deviation are much smaller.

We again correct for inflation and secular trends by applying the HP filter to the deflated time series. Figure 3 displays the result for wheat. It turns out that for the three cereals the annual price deviations are "procyclical", meaning that they are positively correlated to the GDP deviation. The magnitude of the correlation is not large (0.25). Such multicollinearity does not pose a problem for the empirical inference. The price deviation of bacon is not correlated to the GDP deviation.

In addition to the series for GDP, inflation, and food prices we also obtained series on annual average hourly wages for unskilled labor in the urban industrial sector (from Johansen, 1985; see also Mitchell, 2003). The wage deviation in real terms is positively correlated with the business cycle (correlation 0.47 ). Series on the national unemployment rate and on other food prices and fuel (coal, oil) prices are only available for a small subset of later years in our birth observation interval, so we do not use these.

Concerning meteorological data, we obtained time series from the Danish Meteorological Institute on average national annual rainfall and cloud cover in \% (1874-) and temperature (1870-). See Figure 4 for the HP decomposition results for cloud cover. For the missing meteorological observations for year 1873 we assume a cyclical deviation equal to zero.

Finally, the annual national infant mortality rate (IMR) and sex ratio among newborns were obtained from Johansen (1985) and Andreev (2002). The IMR in a given year is the 
period death rate $m_{0}$ for age 0 in that year (see e.g. Andreev, 2002, for details). From the same data sources we also obtain mortality rates for ages higher than 1, but these are an order of magnitude smaller than the IMR and they do not display much variation over time. In our birth-year observation window, the IMR itself is already much lower than in earlier stages of the 19th century. Moreover, it displays a strong downward trend over our window. The transitory IMR component is not related to the business cycle: the correlation is insignificant, and a regression of the latter on the former gives an insignificant coefficient, over 1873-1906 as well as over 1860-1944, whether we allow for autocorrelated errors or not.

The sex ratio is defined as the ratio of the fractions of boys and girls among the newborns in the country in a given year. This variable does not fluctuate wildly over time: in 18731906 it ranges from 1.034 to 1.067 . Nevertheless, it is significantly positively correlated to the business cycle (the correlation coefficient across the years $1873-1906$ is 0.36 ). This is by itself an interesting result. Recessions are often associated with a high stress level, in particular in the late 19th century, as recessions displayed income losses and a high job loss rate in the absence of welfare benefits. Incidentally, note that IMR and sex ratio can be seen as outcomes of deeper early-life conditions like nutritional deficiencies (Almond, Edlund, Li \& Zhang, 2007).

\subsection{Some direct data evidence}

Due to the left-truncation of lifetimes, we cannot simply compare average lifetime durations for those born in booms and those born in recessions, even if we would lump all booms and recessions together and even though we have cyclical indicators that are 
orthogonal to any trends. Specifically, the left-truncation point varies across different birth years. For example, we only observe individuals born in 1873 if their age reaches 70, whereas for those born in 1906 we observe everyone who reaches the age of 37 . To proceed, we compare the mean age $\mathrm{E}\left(T \mid T \geq 73, \tau_{0}\right)$ across different birth years $\tau_{0}$, where $T$ denotes the lifetime duration in years. Specifically, we derive the cyclical component or deviation of the time series of $\mathrm{E}\left(T \mid T \geq 73, \tau_{0}\right)$ in order to remove the trend in longevity, and we correlate this component with the business cycle indicator at $\tau_{0}$. Figure 5 shows the two time series. (The deviation of the conditional mean lifetime is measured such that a value of 0.05 corresponds to 1.35 years.)

For the period 1873-1906, the correlation equals 0.22 . If we omit the year 1877 , which seems to be an outlier in terms of its cyclical indicator, we obtain a correlation of 0.28 which is significantly positive according to a one-sided test at the $5 \%$ level. This provides some evidence that the business cycle at birth has a negative effect on the mortality rate at higher ages. Of course, this result is based on only 33 yearly observations. The test ignores sampling variation within birth years. At the same time, it does not exploit information in the individuals who do not reach the age of 73 or information in higher moments of the distribution of $T$.

Figure 6 displays the Kaplan-Meier estimates of the survival function by whether the business cycle indicator in the birth year is positive or not, conditional on surviving at age 36. Clearly, survival probabilities are lower at every age if born under adverse conditions. 


\section{Estimation of duration models for the individual}

\section{mortality rate}

\subsection{Models for the individual mortality rate}

The individual mortality rate is the natural starting point of the specification of the model, because of our interest in its dependence on conditions early in life. As our model specifications closely follow those in Van den Berg et al. (2006), the present exposition can be brief. Age is measured in days, so we take it to be a continuous random variable. Let $\tau$ denote current calendar time. We may express the mortality rate $\theta$ of an individual at a given point of time in terms of the prevailing age $t$, individual background characteristics $x$, current conditions $z(\tau)$, the indicators $c(\tau-t)$ of conditions early in life, and various interaction terms. For example,

$\log \theta(t \mid x, z, c(\tau-t))=\psi_{1}(t)+\beta^{\prime} x+\alpha^{\prime} c(\tau-t)+\psi_{2} z(\tau)$

where $\alpha$ is the parameter of interest. This is a Proportional Hazards $(\mathrm{PH})$ model with time-varying regressors. The parameter of interest can be estimated with Partial Likelihood Estimation.

Our sample of individuals born in 1873-1906 contains 7540 individuals. The data have barely any individual characteristics. In most analyses we only use the gender. We also allow the mortality rate to depend on a time trend by incorporating a log-linear effect of the birth year. This captures long run secular and period effects. Period effects can also be captured by the residual random variation implicitly modelled in any hazard rate 
model. Van den Berg et al. (2006) find that estimates of long-run effects of early-life conditions do not vary with the modelling of period effects later in life.

The indicators $c(\tau-t)$ of conditions early in life are all measured with a yearly frequency. With only 34 birth years, this implies that we need to restrict attention to model specifications that are parsimonious in terms of the number of indicators. We therefore adopt a strategy in which we consider subsets of indicators separately from each other. We do allow in some cases for interaction terms between $x$ and $c(\tau-t)$.

Note that the sampling scheme entails that we only observe lifetime durations exceeding 36 years. This means that there is no reason to specify a model for durations below 36 years. At the same time, one may argue that selection on survival until age 36 may lead to biased estimates of the effects of early life conditions (see e.g. Vaupel \& Yashin, 1985, for details). But any selection most likely produces a selection of childhood survivors with favorable characteristics among those born in a bad year. So if unobserved heterogeneity is present but is not taken into account then the effects may be biased, but the dynamic selection effect can be expected to generate a positive relation between the cycle during early childhood and observed mortality later in life. So, if we find a negative effect without taking account of unobserved heterogeneity, then the true effect is likely to be at least as negative.

\subsection{Estimation results for models with macro fluctuations at birth}

Table 1 gives the estimation results for the most basic specification where the only indicator of early-life conditions is the cyclical component of the business cycle. The 
estimates concern the mortality rate, so a positive value is associated with a shorter lifetime.

Clearly, the cycle at birth has a significant effect. On average, being born in a recession implies a higher mortality rate later in life. This is in line with the findings in Van den Berg et al. (2006) for the Netherlands for the birth years 1815-1902. To interpret the coefficient, notice that typical high and low values of the cyclical indicator are 0.03 and 0.03 , so that being born in a recession as compared to a boom implies that the mortality rate later in life is $9 \%$ higher. This number is also in line with the findings in Van den Berg et al. (2006). The implied effect on the median lifetime of those who survive until age 36 is about 10 months. The effect is stronger for men than for women. Again, this is in line with the findings in Van den Berg et al. (2006).

The results in Table 1 and those below are robust with respect to changes in the smoothing parameter for the GDP decomposition. They are also robust with respect to the inclusion of additional birth years adjacent to the interval 1873-1906. The PH specification is always confirmed by tests based on Schoenfeld residuals, even in the simple specification of Table 1. Also, interactions effects of cycle and age are insignificant.

Table $\underline{2}$ presents results for the model version in which the year of birth is added as an explanatory variable to account for long-run secular and current effects. The main conclusions are unaffected, although the cyclical effect becomes somewhat smaller. The effect of the squared year of birth is insignificant, but as we add higher order terms, we 
end up with a specification where the estimated year-of-birth polynomial can mimic the effect of the cyclical GDP deviations.

Business cycle effects can be attributed to at least four aspects of early-life conditions: nutrition, disease load, living conditions, and stress. We now perform analyses with other macro indicators, because of their intrinsic relevance as well as in order to see if they can help to understand the business cycle effects.

Food price deviations in the birth year do not have any sizeable or significant effect on the mortality rate later in life. For example, Table $\underline{3}$ presents estimates for a specification where the three cereal price deviations are averaged within years (recall that these are similar). Specifications with only wheat price deviations or other specifications give very similar results.

On average, being born in a year with high cereal prices implies a higher mortality rate later in life, but the effect is insignificant. To interpret the size of the coefficient, notice that typical high and low values of the cyclical indicator are 1 and -1 , so that being born in a year with high cereal prices as compared to a year with low prices implies that the mortality rate later in life is $3.4 \%$ higher. The effect for bacon is even less significant and even smaller (typical high and low values of the cyclical indicator are 0.05 and -0.05 ).

Presumably, average national annual food prices and their deviations are not very informative on the extent to which households face sudden economic hardships. Note that the yearly fluctuations are not dramatically large. Price variation within the year may dominate the variation between years, and price variation within the region might 
dominate national price variation. Perhaps moderate fluctuations at yearly frequencies can be compensated for by way of a temporary substitution towards other food types. An additional complication is that many individuals in the agricultural sector benefit from higher food prices because the latter increase their income.

The fluctuations in the real wage variable in the birth year do not have any effect on the mortality rate later in life. This was to be expected given that the wage variable concerns unskilled work in the urban industrial sector. Probably the wages earned elsewhere in the economy are not strongly correlated to this variable.

Weather deviations in the birth year do not have any sizeable or significant effect on the mortality rate later in life either. For temperature and rain, the weather variation within years strongly dominates the variation between years. Moreover, rainfall is very local, so that there may be a lot of measurement error in the corresponding variable as an indicator for humidity. Table $\underline{4}$ presents estimates for a specification where only cloud cover deviations are included. The results are again robust with respect to the smoothing method.

Clearly, the transitory component of cloud cover at birth has no significant effect. On average, being born in a year with a relatively small amount of direct sunlight implies a higher mortality rate later in life, but the effect is insignificant. To interpret the size of the coefficient, notice that typical high and low values of the cyclical indicator are 3 and -3 , so that being born in a year with low sunshine as compared to a year with high sunshine implies that the mortality rate later in life is about $2 \%$ higher. 
We end this subsection by examining models that include the birth-year deviation of the average annual IMR and the average annual sex ratio among newborns, as explanatory variables for the individual mortality rate. It turns out that both variables have small and insignificant effects. Inclusion of these variables does not influence the results on the business cycle effects. Possibly, the IMR variable is an imperfect indicator of the extent to which babies are exposed to sudden increases in the disease load, in the late 19th century. Moreover, variation within the year may dominate the variation between years, and variation within the region might dominate national variation. We also estimate models in which the mortality rate depends on the IMR deviations in the years prior or after birth and on the deviation of the average national annual mortality rate among infants up to 5 years old. The estimates unambiguously confirm the above conclusions. Finally, concerning the sex ratio it should be noted that the yearly fluctuations are small.

We may conclude from the results so far that among the cohorts and in the country considered, the effect of the business cycle in the birth year on mortality later in life is not explained by yearly fluctuations in national indicators of food prices, wages, weather conditions, infant mortality rates, or sex ratios.

\subsection{The effect of the timing of the birth in the year and the business cycle around the birth year}

In this subsection we estimate models in which the mortality rate later in life depends not only on the business cycle in the birth year but also on the cycle in the year before birth and the years after birth and on the season of birth. This may give some insight into 
whether economic early-life conditions are primarily of importance in utero or at birth or in the years after birth.

First, Table $\underline{5}$ presents estimates for the model including the business cycle in the years after birth. Clearly, the business cycle at the years following the birth year also have a long-run effect on mortality later in life. The magnitude of the effects are smaller than for the business cycle at birth. The cyclical indicator is somewhat autocorrelated over time, so that not too much meaning should be assigned to the magnitude of the individual coefficients.

If we add the business cycle in the year prior to birth then the above results hardly change whereas the coefficient for the year prior to birth is totally insignificant. This suggests that economic conditions during pregnancy are not important for mortality later in life. In fact, we can study this more precisely by taking the date of birth within the birth year into account. Following Doblhammer (2004), we estimate models that include the season of birth as measured in three-monthly intervals. Not surprisingly, the results on the effect of season of birth are in agreement to those in Doblhammer (2004). This effect is generally attributed to seasonal fluctuations in weather conditions and food availability. Subsequently, we interact the season of birth with the business cycle in the year before birth. If the corresponding coefficient for the winter season is largest while the coefficient for the fall season is zero then this would suggest that the economic conditions while in utero are important. However, the results show that all interaction coefficients are very small and are insignificantly different from zero. 
We combine the analyses of 3.2 and 3.3 by estimating a series of models, starting with one in which 20 indicators used so far are included, and subsequently dropping the least significant coefficients. From the start, the business cycle at birth, the spring season, and the time trend are significant, and they remain so until the very end of this horse race.

We end this section by addressing the fact that the data only contain twin pairs. This has not yet been exploited or addressed in this paper. Note that we cannot study intra-pair variation in early life conditions because a pair is born under identical macro conditions. The twin nature of the data does imply that individual observations are not independent if there are unobserved mortality determinants. Estimation with only one individual per twin pair does not affect the point estimates much, although standard errors increase. We also estimate separate models for monozygotic and dizygotic twins. We find that the sensitivity for the business cycle at birth is somewhat larger for monozygotic twins (although it is significant for both groups).

\section{Conclusions}

The business cycle at birth, as measured by the cyclical component of Gross Domestic Product in the birth year, has a significant effect on the mortality rate much later in life, among Danish birth cohorts from 1873-1906. An individual who is born in a recession and who survives until age 35 lives around 10 months shorter than an otherwise identical individual born in a boom. This implies that socio-economic conditions around birth have a causal effect on mortality later in life. We also find that the business cycle in years after 
the birth year affect mortality later in life, whereas the business cycle in the months prior to birth does not seem to have an effect. This suggests that the business cycle effects primarily capture conditions at and after birth. In this sense it complements the effects of birthweight on mortality later in life.

In general, business cycle effects can be attributed to at least four aspects of early-life conditions: nutrition, disease load, living conditions, and stress. We performed analyses with other macro indicators that are linked to one or more of these aspects, in order to see if they can help to understand the business cycle effects. However, these additional analyses mostly led to insignificant effects for the other indicators, while the business cycle effect itself is insensitive to the inclusion of these indicators. We conclude from this that these indicators we used are insufficiently informative as determinants of early-life conditions. There are two possible explanations for this. Consider, in particular, the food price and infant mortality rate. It may be that the fluctuations in these variables in the late 19th century do not reflect damaging effects on the household's nutrition intake or disease exposure. The household may be able to compensate for moderate food price fluctuations by way of a temporary substitution towards other food types, and a high disease load may not be reflected by a high IMR.

The second explanation is that the national and yearly scales in which the other macro indicators are measured may smoothen out too much variation. The fact that the season of birth has the usual significant effect on mortality later in life suggests that this second explanation is more relevant than the first one. A major topic for further research is therefore to obtain macro indicators that display more variation. Notably, they should be 
observed with a higher than yearly frequency and at a lower level of geographical aggregation than the whole country. Food prices and weather indicators are cases in point. Finally, it is useful to be able to distinguish between households that are potentially affected by particular turbulence at the macro level, and those that are not.

To understand business cycle effects it may also be useful to have more information on the living conditions of households. For example, the income and housing conditions of households in crowded and urban quarters may be relatively sensitive to business cycle fluctuations, in comparison to households in the countryside.

\section{Footnotes:}

${ }^{1}$ All time series used in this paper, including descriptions of their origin and/or construction, are available upon request. 


\section{References}

Almond, D.V. (2002), Cohort differences in health: a duration analysis using the National Longitudinal Mortality Study. Working paper, University of Chicago.

Almond, D.V., Edlund, L., Li, H., \& Zhang, J. (2007), Longterm Effects of the 1959-61

China Famine: Mainland China and Hong Kong. Working Paper 13384, NBER.

Andreev, K. (2002), Evolution of the Danish Population from 1835 to 2000. Odense:

University Press of Southern Denmark.

Bengtsson, T., \& Lindström, M. (2000), Childhood misery and disease in later life: The effects on mortality in old age of hazards experienced in early life, southern Sweden, 1760-1894. Population Studies 54, 263-277.

Bengtsson, T., \& Lindström, M. (2003), Airborne infectious diseases during infancy and mortality in later life in southern Sweden, 1766-1894. International Journal of Epidemiology 32, 286-294.

Bengtsson, T., Campbell, C. \& Lee, J. Z. (2004), Life Under Pressure: Mortality and Living Standards in Europe and Asia, 1700-1900. Massachusetts Institute of Technology.

Campbell, C., Lee, J. Z., \& Bengtsson, T, (2004), Economic Stress and Mortality, In T. Bengtsson, C. Campbell \& J. Z. Lee (Eds.), Life under Pressure: Mortality and Living Standards in Europe and Asia, 1700-1900. Massachusetts Institute of Technology. 
Catalano, R. (2002), Economic antecedents of mortality among the very old. Epidemiology 13(2), 133-137.

Catalano, R., Bruckner, T., Hartig, T., \& Ong, M. (2005), Population stress and the Swedish sex ratio. Paediatric and Perinatal Epidemiology 19, 413-420.

Catalano, R., \& Bruckner, T. (2006a), Child mortality and cohort lifespan: a test of diminished entelechy. International Journal of Epidemiology 35(5), 1264-1269.

Catalano, R., \& Bruckner, T. (2006b), Secondary sex ratios and male lifespan: Damaged or culled cohorts. Proceedings of the National Academy of Sciences 103(5), 1639-1643.

Christensen, J. (1985), Landbostatistik Håndbog I dansk landbohistorisk statistik 18301900. Copenhagen: Landbohistorisk Selskab.

Christensen, K., Vaupel, J.W., Holm, N.V. \& Yashin, A.I. (1995), Mortality among twins after age 6: fetal origins hypothesis versus twin method. British Medical Journal 310, $432-436$.

Christensen, K., Wienke, A., Skytthe, A., Holm, N.V., Vaupel, J.W., \& Yashin, A.I. (2001), Cardiovascular mortality in twins and the fetal origins hypothesis. Twin Research $4,344-349$.

Doblhammer, G. (2004), The Late Life Legacy of Very Early Life. Berlin: Springer.

Johansen, H.V. (1985), Dansk Historisk Statistik 1814-1980. Copenhagen: Gyldendal. 
Kannisto, V., Christensen, K., \& Vaupel, J. W. (1997), No Increased Mortality in Later

Life for Cohorts Born During Famine. American Journal of Epidemiology 145(11), 987994.

Lee, J. Z., Campbell, C., \& Bengtsson, T. (2004), New Malthusian Perspectives, In T. Bengtsson, C. Campbell \& J. Z. Lee (Eds.), Life under Pressure: Mortality and Living Standards in Europe and Asia, 1700-1900. Massachusetts Institute of Technology.

Lumey, L., Stein, A. D., Kahn, H. S., van der Pal-de Bruin, K. M., Blauw, G., Zybert, P. A., et al. (2007), Cohort Profile: the Dutch Hunger Winter Families Study. International Journal of Epidemiology 36, 1196-1204.

Løkke, A. (2007), State and insurance: the long-term trends in Danish health policy from 1672 to 1973. Hygiea Internationalis 6, 7-24.

Mitchell, B.R. (2003), International Historical Statistics: Europe, 1750-2000. New York: Palgrave Macmillan.

Moore, S.E., Cole, T.J., Poskitt, E.M.E., Sonko, B.J., Whitehead, R.G., McGregor, I.A., \& Prentice, A.M. (1997), Season of birth predicts mortality in rural Gambia. Nature 388, 434.

Ruhm, C. J. (2000), Are Recessions Good for Your Health? Quarterly Journal of Economics 115(2), 617-650.

Sastry, N. (2004), Trends in socioeconomic inequalities in mortality in developing countries: the case of child survival in Sao Paulo, Brazil. Demography 41, 443-464. 
Sparén, P., Vågerö, D., Shestov, D. B., Plavinskaja, S., Parfenova, N., Hoptiar, V., et al. (2004), Long term mortality after severe starvation during the siege of Leningrad: prospective cohort study. British Medical Journal 328(7430), 11-14.

Statistics Denmark (1958), Landbrugets Priser 1900-1957, Statistiske undersøgelser nr. 1. Copenhagen: Danmarks Statistik.

Stein, Z., Susser, M., Saenger, G., \& Marolla, F. (1975), Famine and Human Development: The Dutch Hunger Winter of 1944-1945. New York: Oxford University Press.

Van den Berg, G.J., Lindeboom, M., \& Portrait, F. (2006), Economic conditions early in life and individual mortality. American Economic Review 96, 290-302.

Van den Berg, G.J., Lindeboom, M., \& López, M. (2007), Inequality in individual mortality and economic conditions earlier in life. Working paper, VU University Amsterdam.

Vaupel, J.W., \& Yashin, A.I. (1985), The deviant dynamics of death in heterogeneous populations, in: N.B. Tuma (ed.), Sociological Methodology 1985. San Francisco: JosseyBass. 


\section{Figure captions:}

Figure 1. Lexis diagram of lifetime observations

Figure 2. Log real GDP per capita: trend and cycle

Figure 3. Yearly mean real wheat price: trend and deviation

Figure 4. Yearly mean cloud cover in \%: trend and deviation

Figure 5. Business cycle and deviation in mean $\mathrm{E}(\mathrm{T} \mid \mathrm{T}>72)$

Figure 6. Non-parametric estimates of the survival function conditional on $\mathrm{T}>36$, by whether the business cycle at birth is $><0$. 


\section{Tables}

Table 1: Parameter estimates of the model for the individual mortality rate, including business cycle indicator at birth

\begin{tabular}{ll}
\hline variable & estimate st.error \\
without interaction gender $\times$ cycle at birth \\
female & $-0.32(0.02)^{*}$ \\
business cycle at birth & $-1.52(0.55)^{*}$ \\
with interaction female $\times$ cycle at birth \\
female \\
business cycle at birth \\
female $\times$ business cycle at birth & $0.86(1.09)$ \\
\end{tabular}

Note: the superindex * represents significance at the $5 \%$ level. 
Table 2: Parameter estimates of the model for the individual mortality rate, including business cycle indicator at birth and the birth year

\begin{tabular}{|lc|}
\hline variable & estimate st.error \\
female & $-0.31(0.02)^{*}$ \\
business cycle at birth & $-1.07(0.55)^{*}$ \\
birth year & $-0.0067(0.0013)^{*}$ \\
& \\
\hline
\end{tabular}

Table 3: Parameter estimates of the model for the individual mortality rate, including business cycle indicator at birth and food price deviations at birth

\begin{tabular}{|lc|}
\hline variable & estimate st.error \\
female & $-0.32(0.02)^{*}$ \\
business cycle at birth & $-1.61(0.55)^{*}$ \\
cyclical cereal price at birth & $0.017(0.013)$ \\
cyclical bacon price at birth & $-0.071(0.19)$ \\
\hline
\end{tabular}

Table 4: Parameter estimates of the model for the individual mortality rate, including business cycle indicator at birth and the transitory component of cloud cover at birth

\begin{tabular}{|lc|}
\hline variable & estimate st.error \\
female & $-0.32(0.02)^{*}$ \\
business cycle at birth & $-1.53(0.55)^{*}$ \\
cyclical cloud cover at birth & $0.0034(0.0048)$ \\
& \\
\hline
\end{tabular}


Table 5: Parameter estimates of the model for the individual mortality rate, including the business cycle indicator in the years after the birth year

$\begin{array}{lc}\text { variable } & \text { estimate st.error } \\ \text { female } & -0.32(0.02)^{*} \\ \text { business cycle in birth year } & -1.41(0.59)^{*} \\ \text { business cycle at age 1 } & -0.85(0.61) \\ \text { business cycle at age 2 } & -0.06(0.60) \\ \text { business cycle at age 3 } & -0.98(0.58)^{*} \\ \text { business cycle at age 4 } & 0.53(0.59)\end{array}$

Note: the superindex * represents one-sided significance for the business cycle coefficents at the $5 \%$ level. 


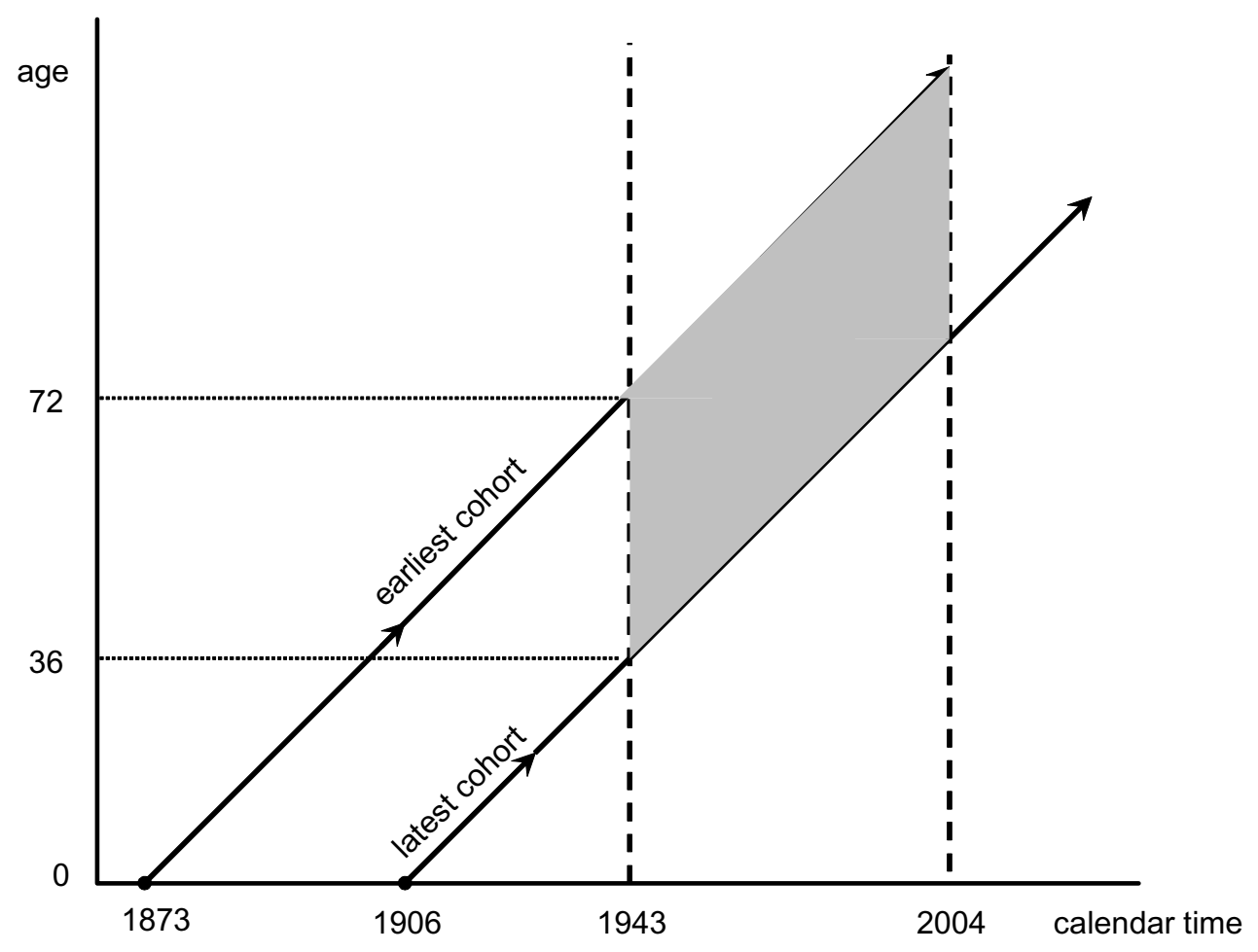

Lexis diagram: grey area covers observed deaths

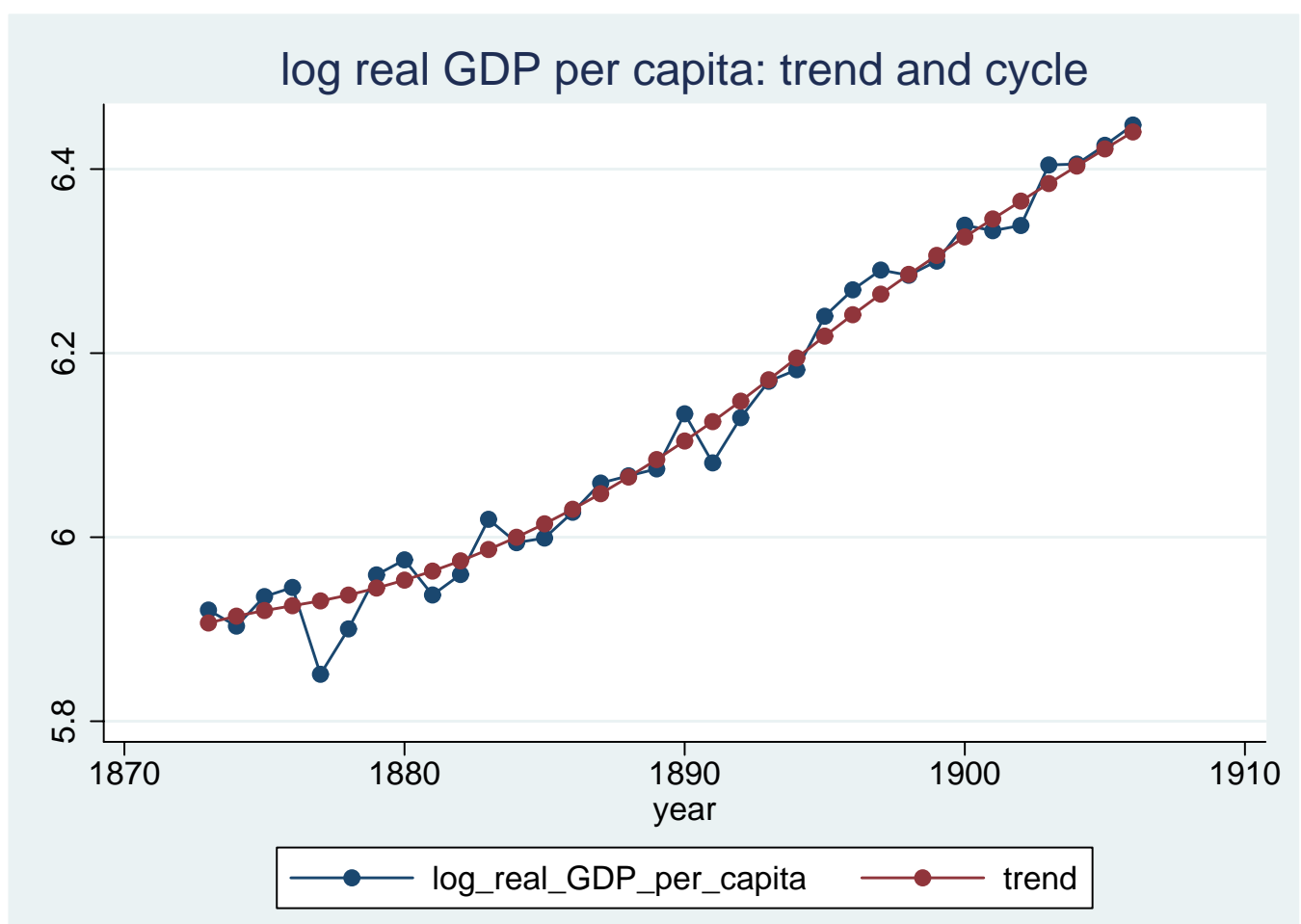




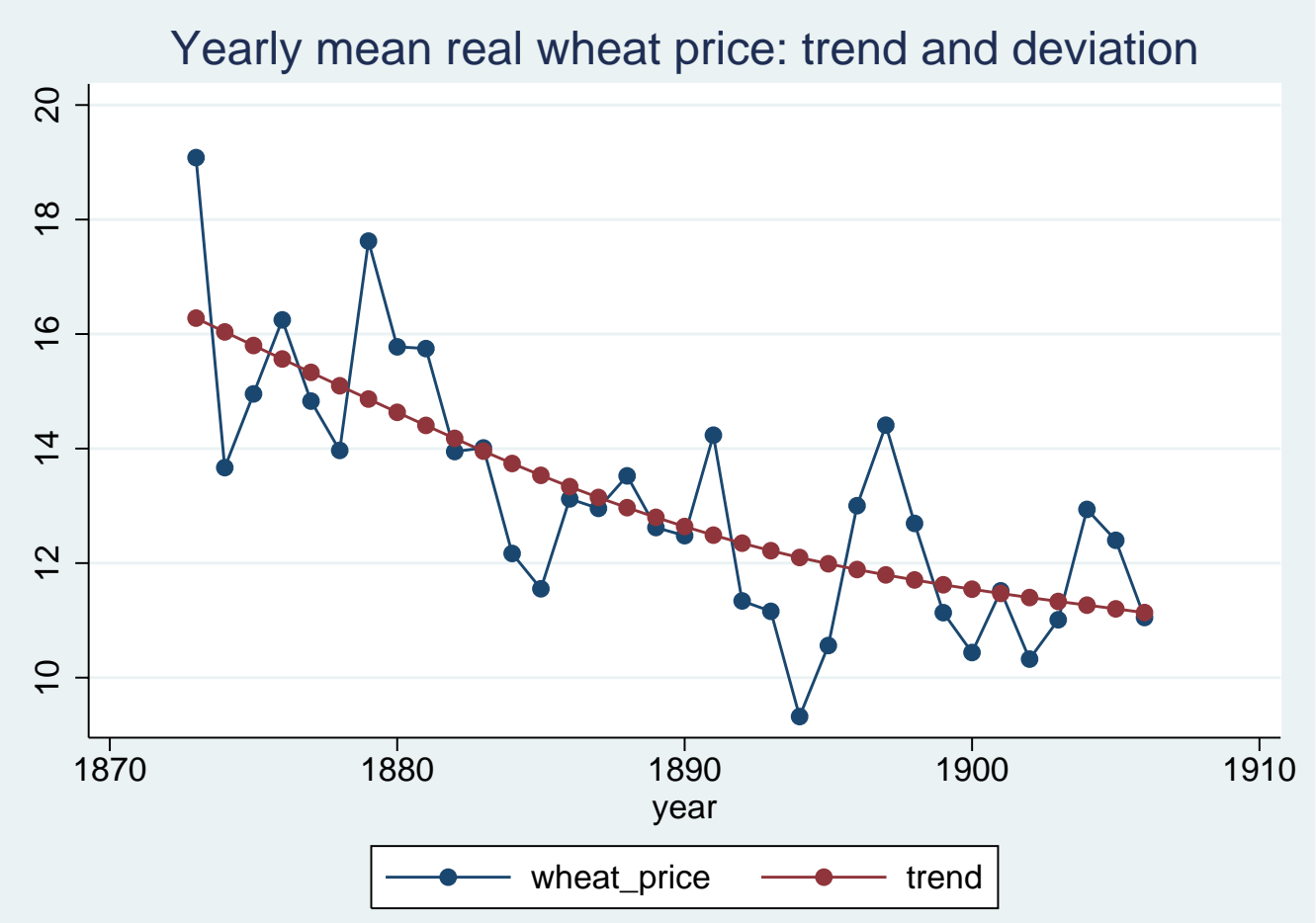

Yearly mean cloud cover in \%: trend and deviation

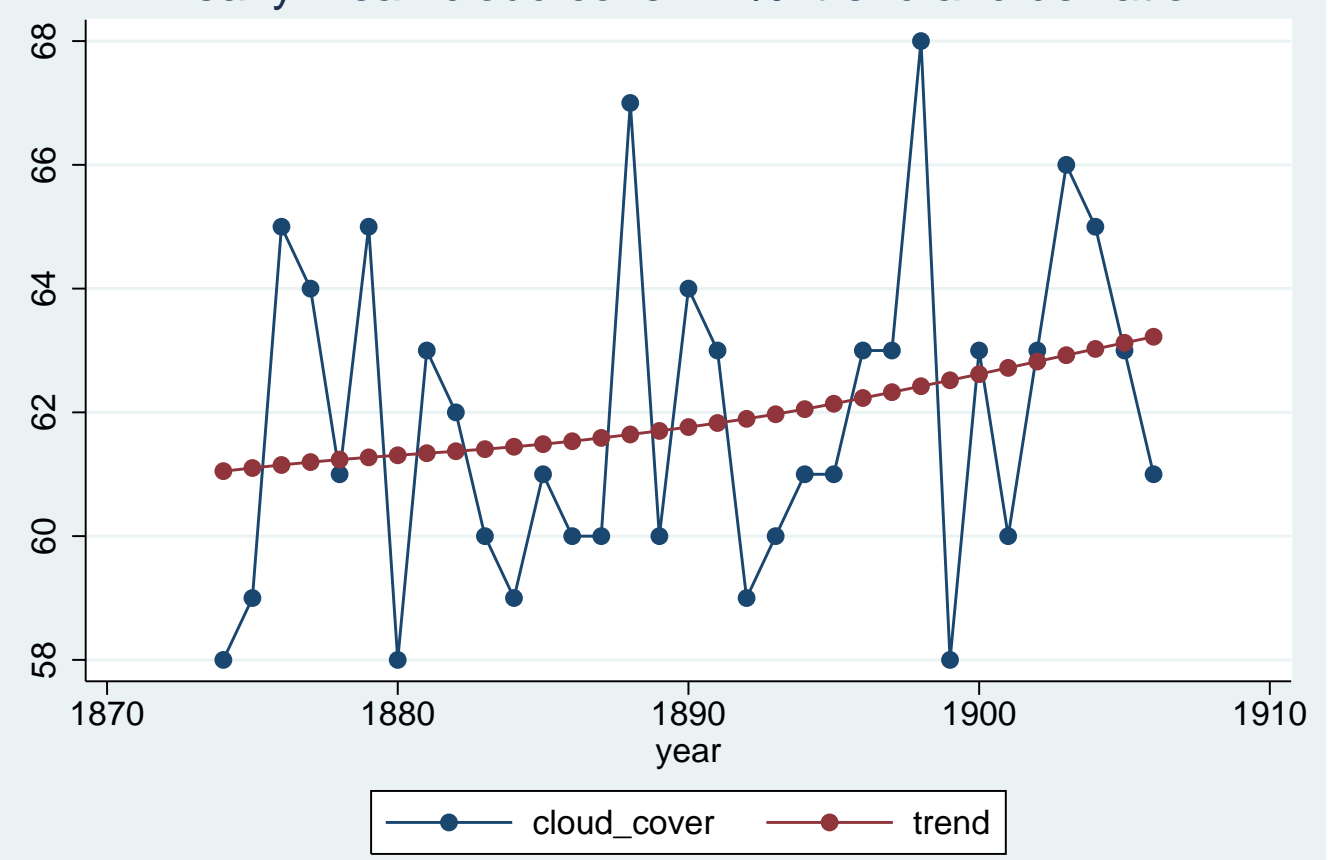




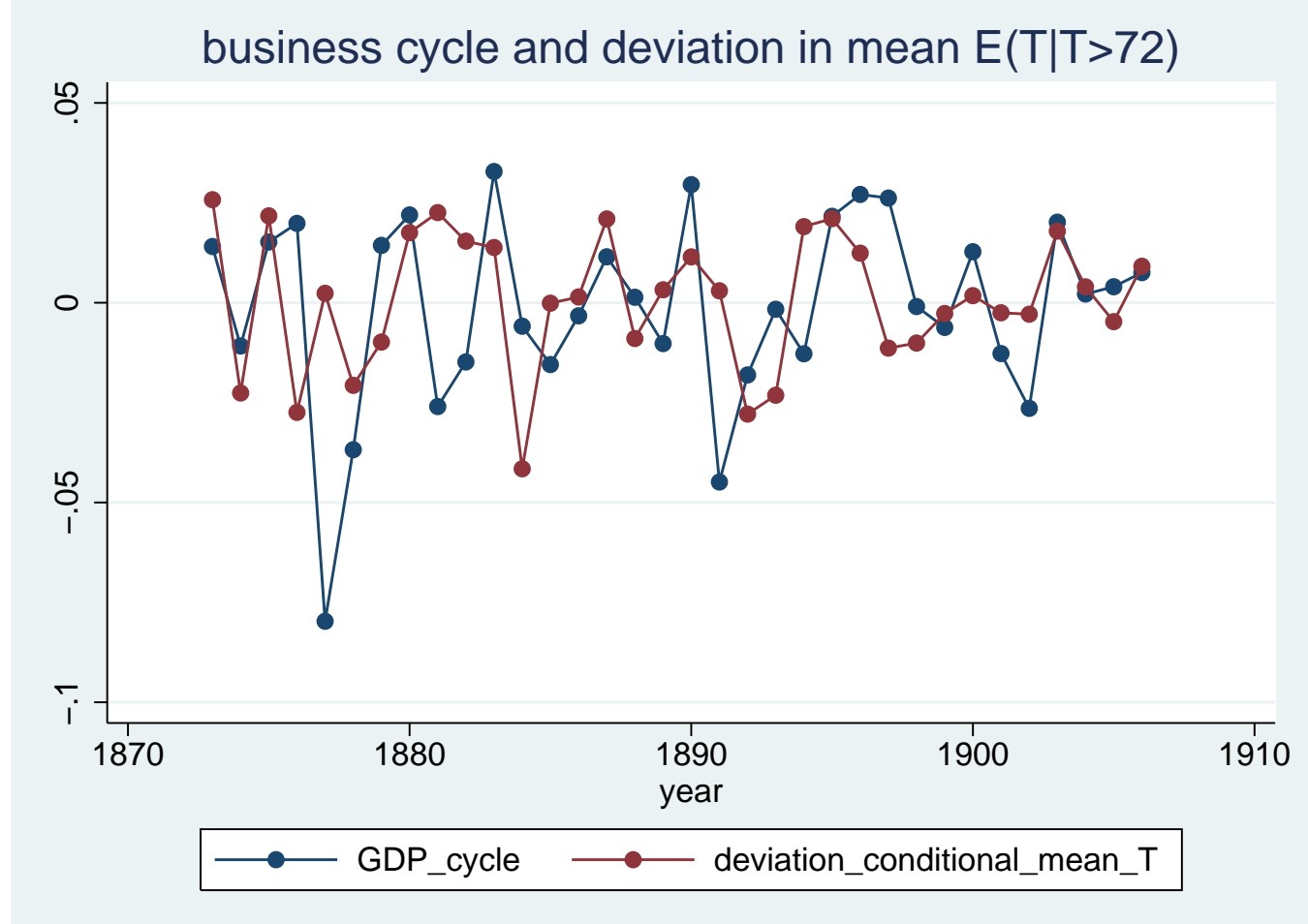

Estimated survivor functions conditional on age $>=36$

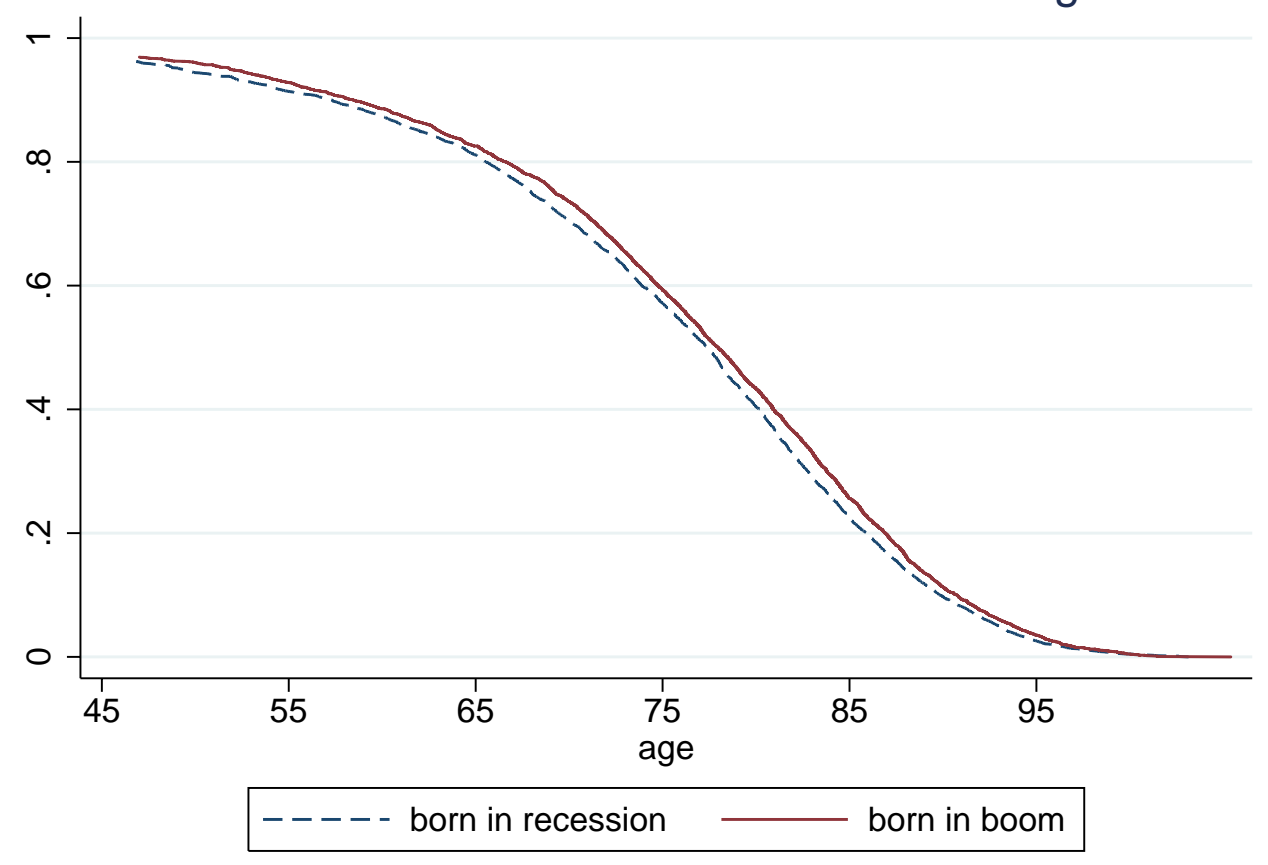

